\title{
Attractors for the Nonclassical Diffusion Equations with Fading Memory and White Noise
}

Haihua Luo, Xianyun $\mathrm{Du}^{*}$

College of Applied Mathematics, Chengdu University of Information Technology, China

DOI: $10.36347 /$ sipms.2020.v07i09.003

| Received: 09.09.2020 | Accepted: 16.09.2020 | Published: 19.09.2020

*Corresponding author: Xianyun Du

\section{Abstract}

In this paper, the longtime behavior of nonclassical diffusion equations with memory decay and white noise is considered. It is proved that the initial value problem has a solution and a random attractor.

Keywords: Nonclassical diffusion equation, Random attractor, White noise.

Copyright @ 2020: This is an open-access article distributed under the terms of the Creative Commons Attribution license which permits unrestricted use, distribution, and reproduction in any medium for non-commercial use (NonCommercial, or CC-BY-NC) provided the original author and source are credited.

\section{INTRODUCTION}

In this paper, we study the long time dynamical behavior of solutions for the following nonclassical diffusion equations with fading memory and white noise:

$$
\left\{\begin{array}{l}
u_{t}-\alpha \Delta u_{t}-\Delta u-\int_{0}^{\infty} k(s) \Delta \mu(t-s) d s=f(u)+\sum_{j=1}^{m} h_{j} d \omega_{j}, \text { in } \Omega, \\
u(x, t)=0, \text { on } \partial \Omega \\
u(x, t)=u_{0}(x, t), x \in \Omega, t \leq 0 .
\end{array}\right.
$$

While $\Omega$ is a bounded domain in $R^{n}(n \geq 3)$. with respect to the stochastic term $\sum_{j=1}^{m} h_{j} d \omega_{j}$,

We do the $O-U$ change for the stochastic term. For the nonlinearity, we presume that $f$ is a Lipschitz continuous function and satisfies:

$$
\begin{aligned}
& \lim _{|s| \rightarrow \infty} \sup \frac{f(s)}{s}<\lambda_{1} . \\
& \left|f^{\prime}(s)\right| \leq C\left(1+|s|^{\frac{4}{n-2}}\right), s \in R . \\
& \left|f^{\prime}(s)\right| \leq C\left(1+|s|^{p}\right) . \forall s \in R .\left\{\begin{array}{l}
p \leq \frac{4}{n-4}, n \geq 5, \\
p \geq 0 .
\end{array}\right.
\end{aligned}
$$

Where $\lambda_{1}$ is the first eigenvalue of $-\Delta$ in $H_{0}^{1}(\Omega)$. and we assume $f(0)=0$.

He effects of fading memory in this equation are shown through the linear time convolution of the function $\Delta u(\cdot)$ and the memory kernel $k(\cdot)$. We assume $k(\cdot) \in C^{2}\left(R^{+}\right), k(s) \leq 0$, and $k^{\prime}(s) \leq 0, \forall s \in R^{+}$. Beside, we also assume that the function $\mu(s)=-k^{\prime}(s)$ and satisfies 


$$
\begin{aligned}
& \mu \in C^{1}\left(R^{+}\right) \cap L^{1}\left(R^{+}\right), \mu(s) \geq 0, \mu^{\prime}(s) \leq 0, \forall s \in R^{+}, \\
& \mu^{\prime}(s)+\delta \mu(s) \leq 0, \forall s \geq 0 .
\end{aligned}
$$

Where $\delta$ is a positive constant? Obviously, it follows the exponential decay of the kernel $k(s)$ and $\mu(s)$ zero. This behavior shows the fading memory of the distant past in the model we will be talking about.

$$
u_{t}-\Delta u=f(u)+g
$$

It does not cover every aspect of reactiondiffusion problems. It ignores the viscosity, elasticity and pressure of the intermediates in the solid diffusion process. In addition, diffuse solid Aifantis was also found. For example, the energy structure equation is

$$
u_{t}-\Delta u_{t}-\Delta u=f(u)+g
$$

In 2010, Xuan Wang in [13] consider viscoelasticity of the conductive medium, that is, the author add fading memory term to $\mathrm{Eq}(1.8)$, the equation

$$
u_{t}-\Delta u_{t}-\Delta u-\int_{0}^{\infty} k(s) \Delta \mu(t-s) d s=f(u)+g(x)
$$

The speed of energy dissipation for Eq (1.9) is faster than for usual nonclassical equation. The conduction of energy is not only affected by present external forces but also by historic external forces.

This equation appear as extension of usual nonclassical diffusion equation in fluid mechanics, solid mechanics and heat conduction theory (see $[1,5,6]$ ).

Since Eq (1.9) contains the term ${ }^{-\Delta u_{t}}$, it is different from the usual reaction diffusion equations with fading memory almost, usually has a fading memory of reaction diffusion equations with high regularity, but for the equation (1.9), if the initial data only belongs to the weak topological space, then the solution is always in the weak topology space, no higher regularity, because of ${ }^{-\Delta u_{t}}$, therefore, for autonomy, cannot use compact Sobolev embedding to verify the key to the solution of semigroup asymptotic compactness.

The long-time behavior of the solution of $\mathrm{Eq}$ (1.8) has been studied for the autonomous case in $[8,9$, $11,12]$. In Y.Xiao [12]. The author has proved the existence of global attractors in $H_{0}^{1}(\Omega)$, when nonlinearity is subcritical and $g(x) \in L^{2}(\Omega)$, In C.Sun, M. Yang [8]. The author have testified the existence of global attractor when nonlinearity is critical and $g(x) \in H^{-1}(\Omega)$. and then Xuan Wang in [23] prove the existence of global attractors in the weak topological
In 1980. Aifantis in [1] point out the classical reaction-diffusion equation

different when the conducting medium is under pressure. Viscoelastic (memory loss). He built up a mathematical model with some concrete examples.It contains the viscosity, elasticity and pressure of the medium, namely, the non-classical diffusion equation:

is the nonclassical diffusion equation with fading memory that is the equation:

space $H_{0}^{1}(\Omega) \times L_{\mu}^{2}\left(R^{+} ; H_{0}^{1}(\Omega)\right)$ and the strong topological space $^{D(\mathrm{~A}) \times L_{\mu}^{2}\left(R^{+} ; D(\mathrm{~A})\right)}$.

Now, if we consider adding a random term of white noise to $\mathrm{Eq}(1.9)$, then the equation is a nonclassical diffusion equation with memory decay and white noise that we will study. Eq (1.1) has not been considered before, and this paper first studies it as a new model to prove the existence of random attractors.Since $\mathrm{Eq}(1.1)$ contains memory terms, we first construct a relatively complex solution space, and do norm inner product in this space. The $O-U$ transformation is applied to the random term.

Attractor is an important concept in the study of asymptotic behavior of deterministic dynamical systems. Crauel, Debussche and Flandoli [15] proposed a general theory for the study of random attractors by defining the attractor set as the attractor of any set of orbitals starting from minus infinity. The random attractor is a compact invariant set, which is dependent on chance and moves with time. The existence of random attractors for two-dimensional random NavierStokes equations is proved by using the theory. In this paper, another method is used to prove the existence of random attractors for the long time behavior of nonclassical diffusion equations with memory decay in white noise. By using the method of operator decomposition (see [19]), the asymptotic compactness of the solution of the system (1.1) is established, which is a key step to obtain the random attractor. 
The arrangement of this article is as follows. The second part gives the relevant concepts and theories. In Section 3, we introduce the OrnsteinOhlenbeck procedure and some properties, and provide some basic Settings for (1.1). In section 4, we prove the existence of a unique random attractor for a stochastic dynamical system generated by (1.1).

\section{Notation and preliminaries}

In this section, we recall some basic notions of the theory of random dynamical system (RDS) (see [14, $15,17,20,21])$ and Kuratowski of non-compactness (see [18]), which is a useful tool to study the attractor (see $[19,22])$.

Let $\left(\mathrm{X},\|\cdot\|_{\mathrm{X}}\right)$ be a separable Banach space with Borel $^{\sigma-a \lg e b r a \mathrm{~B}(\mathrm{X})}$ and $\left(\Omega, F, P\left(\vartheta_{t}\right)_{t \in R}\right) \quad$ be the ergodic metric dynamical system.

Defintion 2.1 [23] A continous random dynamical system over $\left(\Omega, F, P\left(\vartheta_{t}\right)_{t \in R}\right)$ is a $\left(B\left(R^{+}\right)\right) \times F \times B(\mathrm{X})$, $B(X)$ - measurable maping.

$$
S: R^{+} \times \Omega \times X \rightarrow X(t, \omega, x) \rightarrow S(t, \omega, x) .
$$

\section{Satisfying the following properties}

(1) $S(0, \omega, x)=x$ for $\omega \in \Omega$ and $x \in X$;

(2) $S(t+\tau, \omega, \cdot)=S\left(t, \vartheta_{\tau} \omega, \cdot\right) \circ S(\tau, \omega, \cdot)$ for $\tau, \omega \geq 0$, and $\omega \in \Omega$;

(3) $S$ is continous with respect to $x$ for $t \geq 0$ and $\omega \in \Omega$.

A set-vauled $\operatorname{map} B: \Omega \rightarrow 2^{X}$ is called a random closed set if $B(\omega)$ is a nonempty closed set and $\omega \rightarrow d(x, B(\omega))$ is measurable for $x \in X$. A random set $B(\omega)$ is called tempered if for $P-a . s . \omega \in \Omega$ and all $\beta>0$

$$
\lim _{t \rightarrow \infty} e^{-\beta t} \sup \left\{\|b\|_{X}: b \in B\left(\vartheta_{-t} \omega\right)\right\}=0
$$

Let $D$ be the collection of all tempered random subsets in $X$ and $\{K(\omega)\}_{\omega \in \Omega} \in D$. Then $\{K(\omega)\}_{\omega \in \Omega}$ is called a random absorbing set for $S$ in $D$ if for $B(\omega) \in D$ and $P$ - a.e. $\omega \in \Omega$, there exist $t_{B}(\omega)>0$ such that

$$
S\left(t, \vartheta_{-t} \omega, B\left(\vartheta_{-t} \omega\right)\right) \subset K(\omega) \text { for all } t \geq t_{B}(\omega)
$$

Definition 2.2 [23] A random set $\{\mathrm{A}(\omega)\} \in D$ is random attractor (or pullback attractor) for a $R D S S$ if the following conditions are satisfied, for $P$-a.s. $\omega \in \Omega$,

(i) $A(\omega)$ is a random compact set.i.e. $\omega \rightarrow d(x, A(\omega))$ is measurable for every $x \in X$ and $A(\omega)$ is compact;

(ii) $\{A(\omega)\}$ is strictly invariant, $i, e$.

$$
S(t, \omega, A(\omega))=A\left(\vartheta_{t} \omega\right) \text { for all } t \geq 0 ;
$$

(iii) $\{A(\omega)\}$ attracts every set in $D, i$. e. for all $B=\{B(\omega)\} \in D$,

$$
\lim _{t \rightarrow \infty} d_{H}\left(\varphi\left(t, \vartheta_{-t}(\omega)\right), B\left(\vartheta_{-t} \omega\right), A(\omega)\right)=0 ;
$$

Where $d_{H}$ is the Hausdorff semi-distance.

Let $B$ be a bounded set is a Banach space $X$.The Kuratowski measure of non-compactness $\alpha(B)$ of $B$ is defined by

$$
\alpha(B)=\inf \{d>0: B \text { admits a finite cover by sets of diameter } \leq d\} .
$$

We define $\alpha(B)=\infty$, if $B$ is unbounded, see [8].

Definition 2.3. [19] A random dynamical system $S$ on a Polish space $(X, d)$ is almost surely $D-\alpha-$ contracting if

$$
\lim _{t \rightarrow \infty} \alpha\left(S\left(t, \vartheta_{-t} \omega, A\left(\vartheta_{-t} \omega\right)\right)\right)=0 \text { for } A \in D
$$


Lemma 2.4. For a random dynamical system $S(t, \omega)$ on a separabal Banach space $\left(X,\|\cdot\|_{X}\right)$, if almost surely the following hold:

(1) $S(t, \omega)=S_{1}(t, \omega)+S_{2}(t, \omega)$;

(2) For any tempered random variable $a \geq 0$, there exist $r(a)(0 \leq r \leq \infty)$, a.s. such that for the closed ball $B_{a}$ with radius $a$ in $X, S_{1}\left(t, \vartheta_{-t} \omega, B_{a}\left(\vartheta_{-t} \omega\right)\right)$ is precompact in $X$ for all $t>r(a)$.

(3) $\left\|S_{2}\left(t, \vartheta_{-t} \omega, u\right)\right\|_{X} \leq K\left(t, \vartheta_{-t} \omega, a\right), t>0, u \in B_{a}(\omega)$ and $K(t, \omega, a)$ is a measurable function with respect to $(t, \omega, x)$ which satisfies

$$
\lim _{t \rightarrow \infty} K\left(t, \vartheta_{-t} \omega, a\right)=0
$$

Then $S(t, \omega)$ is almost surely $D-\alpha-$ contracting ( see [19]).

Lemma 2.5. Let $S(t, \omega)$ be a random dynamical system on a Polish space $\left(X,\|\|_{X}\right)$.

Assume that

(1) $S(t, \omega)$ has an absorbing set $B(\omega) \in D$;

(2) $S(t, \omega)$ is almost surely $D-\alpha$-contracting .

Then $S(t, \omega)$ possesses a global random attractor in $X$.

\section{The basic setting}

Now, we consider the one-dimensional Ornstein-Uhlenbeck equation

$$
d z_{j}+\lambda z_{j} d t=d \omega_{j}(t)
$$

So

$$
z_{j}(t)=z_{j}\left(\vartheta_{t} \omega_{j}\right)=-\lambda \int_{-\infty}^{0} e^{\lambda \tau}(\tau) d \tau, \text { for } t \in R
$$

Putting $z\left(\vartheta_{t} \omega\right)=\sum_{j=1}^{m}(I-\alpha \Delta)^{-1} h_{j} z_{j}\left(\vartheta_{t} \omega_{j}\right)$, where $\Delta i s$ the Laplacian with domain $H_{0}^{1} \cap H^{2}(0, l)$, By (3.1) we have

$$
d z-\alpha d(\Delta z)+\alpha(z-\alpha z) d t=\sum_{j=1}^{m} h_{j} d \omega_{j}
$$

Lemma 3.1. [23] For $\hbar>0$, there exist a tempered random variable $\rho_{1}: \Omega \rightarrow R$ such that

$$
\left\|z\left(\vartheta_{t} \omega\right)\right\|_{2 p+2} \leq e^{\lambda|t|} \rho_{1}(\omega) \text { for } t \in R \text { and } \omega \in \Omega
$$

Where $\rho \geq 0$ and $\rho_{1}(\omega), \omega \in \Omega$ satisfies:

$$
\rho_{1}\left(\vartheta_{t} \omega\right) \leq e^{\lambda|t|} \rho_{1}(\omega) \text { for } t \in R
$$

Corollary 3.2. [23] For $\hbar>0$, there exists a tempered random variable $\rho_{2}: \Omega \rightarrow R$ such that for $\hbar=0$ or 1 ,

$$
\left\|A^{\frac{\hbar}{2}} z\left(\vartheta_{t} \omega\right)\right\|+\alpha \sqrt{\lambda_{1}}\left\|A^{\frac{1+\hbar \hbar}{2}} z\left(\vartheta_{t} \omega\right)\right\| \leq e^{\bar{\lambda}|t|} \rho_{2}(\omega) .
$$

For $t \in R$ and $\omega \in \Omega$, where $\lambda_{1}$ is the first eigenvalue of $-\Delta$.

As in [3], we introduce a new variable which reflect the past history of the Eq (1.1), that is,

$$
\eta^{t}(x, s)=\int_{0}^{s} u(x, t-r) d r, s \geq 0,
$$


Then

$$
\partial_{t} \eta^{t}(x, s)=u(x, t)-\partial_{s} \eta^{t}(x, s), s \geq 0
$$

In view of assumption about memory kernel $\mu(\cdot)$, let $L_{\mu}^{2}\left(R^{+} ; H_{r}\right)$ be the family of Hilbert space of function $\varphi: R^{+} \rightarrow H_{r}, 0<r<3$, endowed with the inner product and norm, respectively

$$
\begin{aligned}
\left\langle\varphi_{1}, \varphi_{2}\right\rangle_{\mu, H_{r}} & =\int_{0}^{\infty} \mu(s)\left\langle\varphi_{1}, \varphi_{2}\right\rangle_{H_{r}} d s . \\
\|\varphi\|_{\mu, H_{r}} & =\int_{0}^{\infty} \mu(s)\|\varphi(s)\|_{H_{r}}^{2} d s .
\end{aligned}
$$

\section{Now we introduce the family of Hilbert spaces}

$$
M_{r}=H_{r} \times L_{\mu}^{2}\left(R^{2} ; H_{r}\right)
$$

\section{And endowed norm}

$$
\|z\|_{M_{r}}=\left\|\left(u, \eta^{t}\right)\right\|_{M_{r}}=\left(\frac{1}{2}\|u\|_{H_{r}}^{2}+\left\|\eta^{t}\right\|_{\mu, H_{r}}^{2}\right)^{\frac{1}{2}} .
$$

In order to estimate conveniently, we first show the preliminary result as follows (cf. $[2,4,7])$.

Lemma 3.3. [13] Setting, Let memory kernel satisfy, then for any, there exist a constant, such that:

$$
\left\langle\eta^{t}, \eta_{s}^{t}\right\rangle_{\mu, H_{r}} \geq \frac{\delta}{2}\left\|\eta^{t}\right\|_{\mu, H_{r}}^{2}
$$

We also need the following results to prove the asymptotic compactness about memory term as well as the existence of global attractors.

Lemma 3.4. (See $[2,4,7])$ Assuming that $\mu \in C^{1}\left(R^{+}\right) \cap L^{1}\left(R^{+}\right)$is a nonnegative function, and satisfies: if the exist $s_{0} \in R^{+}$, such that $\mu\left(s_{0}\right)=0$, then $\mu(s)=0$ for all $s \geq s_{0}$ holds.

Moreover, Let $B_{0}, B_{1}, B_{2}$ be Banach

space, here $B_{0}, B_{1}$ are reflexive and satisfy $\quad B_{0} \rightarrow B_{1} \rightarrow B_{2}$.

Where the embedding $B_{0} \rightarrow B_{1}$ is compact, let $C \subset L_{\mu}^{2}\left(R^{+} ; B_{1}\right)$ satisfy

(i) $C$ in $L_{\mu}^{2}\left(R^{+} ; B_{0}\right) \cap H_{\mu}^{1}\left(R^{+} ; B_{2}\right)$;

(ii) $\sup _{\eta \in C}\|\eta(s)\|_{B_{1}}^{2} \leq \hbar(s), \forall s \in R^{+}, \hbar(s) \in L_{\mu}^{1}\left(R^{+}\right)$;

Then $C$ is relatively compact in $L_{\mu}^{2}\left(R^{+} ; B_{1}\right)$.

Lemma 3.5. (See [17]) Let $H$ be a complete metric space, $\{S(t)\}_{t \geq 0}$ be a semigroup in and has a bounded absorbing set $B_{0}$ in $H_{0}$. If for every $t \geq 0$, the operator $S(t)$ allows the decomposition $S(t)=S_{1}(t)+S_{2}(t)$, and satisfies:

(i) The semigroup $\left\{S_{1}(t)\right\}_{t \geq 0}$ is uniformly compact,as $t$ is increasing sufficiently;

(ii) The operator $S_{2}(t): H \rightarrow H$ is continuous, and for any bounded set $B \subset H$, as $t \rightarrow \infty$

$$
r_{B}(t)=\sup _{\varphi \in B}\left\|S_{2}(t) \varphi\right\|_{H} \rightarrow 0
$$

Then $\omega$-limit set of absorbing set is global attractors of $\{S(t)\}_{t \geq 0}$. 
Let $v(t, \omega)=u(t, \omega)-z\left(\vartheta_{t} \omega\right)$. Then setting $\mu(s)=-k^{\prime}(s)$ and using assumption $k(\infty)=0$, Eq (1.1) can be transfored into the following system:

$$
\begin{aligned}
& v_{t}-\alpha \Delta v_{t}-\Delta v-\int_{0}^{\infty} \mu(s) \Delta \eta(s) d s=f\left(v+z\left(\vartheta_{t} \omega\right)\right)+\left(1-\alpha^{2}\right) \Delta z\left(\vartheta_{t} \omega\right)+\alpha z\left(\vartheta_{t} \omega\right) \\
& v(x, t)=0 \quad x \in \partial \Omega, t \geq 0 \\
& v(x, 0)=v_{0}(x), x \in \Omega \\
& \eta^{t}(x, s)=0,(x, s) \in \partial \Omega \times R^{+}, t \geq 0
\end{aligned}
$$

We set $E_{0}=H_{0}^{1} \times L^{2}(0, l), E_{1}=H^{2} \cap H_{0}^{1} \times H_{0}^{1} \cap L^{2}(0, l)$. Then $E_{1} \rightarrow E_{0}$ with compact imbedding.

By a Galerkin method as in [10], it can be proved that under assumptions (1.4), for $P-$ a.e. $\omega \in \Omega$ and for every $\left(v_{0}, \eta^{0}\right) \in E_{0}$, problem(3.6)-(3.9) have a unique solution $v \in C\left(R^{+}, E_{0}\right)$ and the solution $(v)$ is continuous with respect to . Hence, the solution mapping generates a RDS. It is called stochastic flow associated with the nonlinear strain wave equation with additive noise.

\section{Uniform time a priori estimates and random attractors}

Let $E_{0}=H_{0}^{1} \times L^{2}(0, l)$ endowed with the inner product and norm $\left(Y_{1}, Y_{2}\right)_{E_{0}}=v_{H_{0}^{1}}+\eta_{L_{\mu}^{2}}^{t}$,

$\|Y\|_{E_{0}}=\|v\|_{H_{0}^{1}}+\left\|\eta^{t}\right\|_{L_{\mu}^{2}}, Y_{j}=\left(v_{j}, \eta^{t}\right), Y_{0}=\left(v_{0}, \eta^{0}\right)$. We define the following unifor estimates in $E_{0}$.

Lemma 4.1. Suppose that $f$ satisfies (1.2)-(1.4). Then for $B=\{B(\omega)\}_{\omega \in \Omega} \in D \quad Y_{0}=\left(v_{0}, \eta^{0}\right) \in B(\omega)$, and for $P-$ a.e. $\omega \in \Omega$, there exists $T=T(B, \omega)>0$, such that

$$
\left\|Y\left(t, \vartheta_{-t} \omega, Y_{0}\left(\vartheta_{-t} \omega\right)\right)\right\|_{E_{0}} \leq R(\omega) \text { for } t \geq T,
$$

Where $R(\omega)=c\left(1+\rho_{1}^{2 p+2}(\omega)+\rho_{2}^{2}(\omega)\right)$ is a positive random function.

Proof. Taking the inner product of (3.8) with $v$ and using $v=u-z\left(\vartheta_{t} \omega\right)$, we have

$$
\frac{d}{d t} \phi_{0}(t, \omega)+H_{0}(t, \omega)=0 \text {, }
$$

Where

$$
\begin{aligned}
& \phi_{0}(t, \omega)=\frac{1}{2}\left(\|v\|^{2}+\alpha\|\nabla v\|^{2}+\left\|\eta^{t}\right\|_{\mu, E_{0}}^{2}\right), \\
& H_{0}(t, \omega)=\|\nabla v\|^{2}+\left\langle\eta^{t}(s), \eta_{s}^{t}(s)\right\rangle_{\mu, E_{0}}-\left\langle f\left(v+z\left(\vartheta_{t} \omega\right)\right), v\right\rangle \\
& -\left\langle\left(1-\alpha^{2}\right) \nabla z\left(\vartheta_{t} \omega\right), v\right\rangle-\left\langle\alpha z\left(\vartheta_{t} \omega\right), v\right\rangle .
\end{aligned}
$$

In condition of (1.2)-(1.4), we have

$$
\begin{gathered}
\left\langle f\left(v+z\left(\vartheta_{t} \omega\right)\right), v\right\rangle \leq \lambda_{1}\left\|v+z\left(\vartheta_{t} \omega\right)\right\|^{2}-w\|v\|^{2}+c \\
\leq \frac{3\left(\lambda_{1}-w\right)}{2}\|v\|^{2}+\frac{\lambda_{1}-w}{2}\left\|z\left(\vartheta_{t} \omega\right)\right\|^{2}+c \\
\leq\left(1-\frac{w}{\lambda_{1}}\right)\|\nabla v\|^{2}+\frac{\lambda_{1}-\omega}{3}\left\|z\left(\vartheta_{t} \omega\right)\right\|^{2}+c .
\end{gathered}
$$

Here $w>0$, and

$$
\left\langle\left(1-\alpha^{2}\right) \nabla z\left(\vartheta_{t} \omega\right), v\right\rangle+\left\langle\alpha z\left(\vartheta_{t} \omega\right), v\right\rangle
$$




$$
\leq \frac{w}{4 \lambda_{1}}\|\nabla v\|^{2}+\frac{\lambda_{1}\left(1-\alpha^{2}\right)}{2 w}\left\|\nabla z\left(\vartheta_{t} \omega\right)\right\|^{2}+\frac{\lambda_{1} \alpha}{2 w}\left\|z\left(\vartheta_{t} \omega\right)\right\|^{2}
$$

Apply lemma (3.3), we know

$$
\left\langle\eta^{t}(s), \eta_{s}^{t}(s)\right\rangle_{\mu, E_{0}} \geq \frac{\delta}{2}\left\|\eta^{t}\right\|_{\mu, E_{0}}^{2} .
$$

Then, we get

$$
\frac{1}{2} \frac{d}{d t}\left(\|v\|^{2}+\alpha\|\nabla v\|^{2}+\left\|\eta^{t}\right\|_{\mu, E_{0}}^{2}\right)+\frac{\sigma}{2}\left(\|v\|^{2}+\alpha\|\nabla v\|^{2}+\left\|\eta^{t}\right\|_{\mu, E_{0}}^{2}\right) \leq c_{1}\left(1+p_{0}\left(\vartheta_{t} \omega\right)\right) .
$$

Where

$$
\sigma=\min \left\{\frac{w}{2}, \frac{w}{2 \lambda_{1} \alpha}, \delta\right\} .
$$

And

Obviously,

$$
p_{0}\left(\vartheta_{t} \omega\right)=\left\|\nabla z\left(\vartheta_{t} \omega\right)\right\|^{2}+\left\|z\left(\vartheta_{t} \omega\right)\right\|^{2}
$$

$$
\|Y(t)\|_{E_{0}}^{2} \leq \phi_{0}(t, \omega) \leq c_{0}\|Y(t)\|_{E_{2}}^{2} .
$$

According to Gronwall lemma,

$$
\|Y(t)\|_{E_{0}}^{2} \leq c_{0}\|Y(0)\|_{E_{0}}^{2} e^{-\sigma t}+\frac{c_{1}}{\sigma} p_{0}\left(\vartheta_{t} \omega\right) .
$$

Hence, by (4.1) and (4.6), we have

$$
\frac{d}{d t} \phi_{0}(t, \omega)+\lambda \phi_{0}(t, \omega) \leq c_{1} p_{0}\left(\vartheta_{t} \omega\right)+c_{1},
$$

Where $\lambda=\frac{\sigma}{2}>0$, By lemma 3.1. with $\lambda=\frac{\lambda}{4 p+4}$ and Corollary 3.2. with $\lambda=\frac{\lambda}{4}$, for $P-$ a.e. $\omega \in \Omega$ and $t \in R$, we obtain:

$$
p_{0}\left(\vartheta_{t} \omega\right) \leq 2 e^{\frac{1}{2} \lambda|t|}\left(\frac{1}{2} \rho_{1}^{2 p+2}(\omega)+\frac{1}{2} \rho_{2}^{2}(\omega)\right)
$$

It follows from (4.9) that for all $t \geq 0$

$$
\phi_{0}(t, \omega) \leq e^{-\lambda t} \phi_{0}(0, \omega)+c_{1} \int_{0}^{t} e^{\lambda(\tau-t)} p_{0}\left(\vartheta_{\tau} \omega\right) d \tau+\frac{c_{1}}{\lambda} .
$$

Replacing $\omega$ by $\vartheta_{-t} \omega$ with $t \geq 0$ in (4.11) and using (4.10), we get:

$$
\begin{gathered}
\phi_{0}(t, \omega) \leq e^{-\lambda t} \phi_{0}\left(0, \vartheta_{-t} \omega\right)+c_{1} \int_{0}^{t} e^{\lambda(\tau-t)} p_{0}\left(\vartheta_{\tau-t} \omega\right) d \tau+\frac{c_{1}}{\lambda} \\
\leq e^{-\lambda t} \phi_{0}\left(0, \vartheta_{-t} \omega\right)+c_{1} \int_{-t}^{0} e^{\lambda \tau} p_{0}\left(\vartheta_{\tau} \omega\right) d \tau+\frac{c_{1}}{\lambda} \\
\leq e^{-\lambda t} \phi_{0}\left(0, \vartheta_{-t} \omega\right)+c_{1} \int_{-t}^{0} e^{\frac{1}{2} \lambda \tau}\left(\rho_{1}^{2 p+2}(\omega)+\rho_{2}^{2}(\omega)\right) d \tau+\frac{c_{1}}{\lambda} \\
\leq e^{-\lambda t} \phi_{0}\left(0, \vartheta_{-t} \omega\right)+c_{2}\left(1+\rho_{1}^{2 p+2}(\omega)+\rho_{2}^{2}(\omega)\right),
\end{gathered}
$$

Where $c_{2}=\frac{3 c_{1}}{\lambda}$ is a deterministic positive constant. This together with (4.8) show that

$$
\| Y\left(t, \vartheta_{-t} \omega, Y_{0}\left(\vartheta_{-t} \omega\right)\left\|_{E_{0}}^{2} \leq c_{0}\right\| Y\left(\vartheta_{-t} \omega\right) \|_{E_{0}}^{2} e^{-\sigma t}+c_{*}\left(1+\rho_{1}^{2 p+2}(\omega)+\rho_{2}^{2}(\omega)\right),\right.
$$


Where $c_{*}=\frac{c_{1}\left(1+e^{-\lambda t}\right)}{\sigma}$ is a deteminstic positive constant.

Denote

$$
K(\omega)=\left\{Y \in E_{0}:\|Y\|_{E_{0}} \leq R(\omega)\right\}
$$

Then $\{K(\omega)\}_{\omega \in \Omega} \in D$ is an absorbing set in $E_{0}$.

In order to prove that $R D S S(t, \omega)$ is almost surely $D$ - $\alpha$-contracting on $E_{0}$ by Lemma 2.5 . we decompose the solution $Y=\left(v, \eta^{t}\right)$ of the (3.6)-(3.9) with the initial value $Y_{0}=\left(v_{0}, \eta^{0}\right)$ into two part. Define by $Y\left(v^{a}, \eta^{t}\right)=S_{1}(t)\left(v_{0}, \eta^{0}\right)$ is the solution of the equations

$$
v_{t}^{a}-\alpha \Delta v_{t}^{a}-\Delta v^{a}-\int_{0}^{\infty} \mu(s) \Delta \eta(s) d s=f\left(v^{a}+z\left(\vartheta_{t} \omega\right)\right) .
$$

With $\left.\left(v^{a}, \eta^{t}\right)\right|_{t=0}=\left(v_{0}, \eta^{0}\right)$ and homogeneous boundary condition. Then

$$
Y^{b}=\left(v^{b}, \eta^{t}\right)=S_{2}(t)\left(v_{0}, \eta^{0}\right)=S(t)\left(v_{0}, \eta^{0}\right)-S_{1}(t)\left(v_{0}, \eta^{0}\right),
$$

Is the solution of the problems

$$
v_{t}^{b}-\alpha \Delta v_{t}^{b}-\Delta v^{b}-\int_{0}^{\infty} \mu(s) \Delta \eta(s) d s=f\left(v+z\left(\vartheta_{t} \omega\right)\right)+\left(1-\alpha^{2}\right) \Delta z\left(\vartheta_{t} \omega\right)+\alpha z\left(\vartheta_{t} \omega\right),
$$

With the initial data $\left.\left(v^{b}, \eta^{t}\right)\right|_{t=0}=(0,0)$ and homogeneous boundary conditions.

Lemma 4.2. Assume $Y_{0}=\left(v_{0}, \eta^{0}\right) \in B(\omega) \in D$, and (1.2)-(1.4) hold, Then

$$
\left\|Y^{a}\left(t, \vartheta_{-t} \omega, Y_{0}\left(\vartheta_{-t} \omega\right)\right)\right\|_{E_{0}}^{2} \leq C\left\|Y_{0}\left(\vartheta_{-t} \omega\right)\right\|_{E_{0}}^{2} e^{-\lambda_{2} t}
$$

Proof Taking the inner product of (3.8) with $v^{a}$ and using $v^{a}=u^{a}-z\left(\vartheta_{t} \omega\right)$, we have

$$
\frac{d}{d t} \phi_{1}(t, \omega)+H_{1}(t, \omega)=0
$$

Where

$$
\phi_{1}(t, \omega)=\frac{1}{2}\left(\left\|v^{a}\right\|^{2}+\alpha\left\|\nabla v^{a}\right\|^{2}+\left\|\eta^{t}\right\|_{\mu, E_{0}}^{2}\right),
$$

And

$$
H_{1}(t, \omega)=\left\|\nabla v^{a}\right\|^{2}+\left\langle\eta^{t}(s), \eta_{s}^{t}(s)\right\rangle_{\mu, E_{0}} \cdot
$$

Hence, we get

$$
\frac{1}{2} \frac{d}{d t}\left(\left\|v^{a}\right\|^{2}+\alpha\left\|\nabla v^{a}\right\|^{2}+\left\|\eta^{t}\right\|_{\mu, E_{0}}^{2}\right)+\left\|\nabla v^{a}\right\|^{2}+\left\langle\eta^{t}(s), \eta_{s}^{t}(s)\right\rangle_{\mu, E_{0}}=0 .
$$

By using Lemma 3.3.and $\lambda_{1}$ is the first eigenvalue of $-\Delta$ we have

$$
\frac{1}{2} \frac{d}{d t}\left(\left\|v^{a}\right\|^{2}+\alpha\left\|\nabla v^{a}\right\|^{2}+\left\|\eta^{t}\right\|_{\mu, E_{0}}^{2}\right)+\lambda\left(\left\|v^{a}\right\|^{2}+\alpha\left\|\nabla v^{a}\right\|^{2}+\left\|\eta^{t}\right\|_{\mu, E_{0}}\right) \leq 0 .
$$

So, we obtain

$$
\frac{d}{d t} \phi_{1}(t, \omega)+\lambda_{2} \phi_{1}(t, \omega) \leq 0
$$

Where $\lambda_{2}=\min \left\{w, \frac{w}{\lambda_{1} \alpha}, \delta\right\}$. Applying Gronwall's lemma, we botain for all $t \geq 0$

$$
\phi_{1}(t, \omega) \leq \phi_{1}(0, \omega) e^{-\lambda_{2} t}
$$


By arguments similar to (4.7), we can get derive that

$$
\left\|Y^{a}\left(t, \omega, Y_{0}(\omega)\right)\right\|_{E_{0}}^{2} \leq c\left\|Y_{0}(\omega)\right\|_{E_{0}}^{2} e^{-\lambda_{2} t}
$$

Replacing $\omega$ by $\vartheta_{-t} \omega$ with $t \geq 0$ in (4.17), implies that the result hold.

Lemma 4.3. Assume $Y_{0}=\left(v_{0}, \eta^{0}\right) \in B(\omega) \in D$ and (1.4) hold, Then

$$
\left\|Y^{b}\left(t, \vartheta_{-t} \omega, Y_{0}\left(\vartheta_{-t} \omega\right)\right)\right\|_{E_{0}}^{2} \leq 2 R_{*}^{2}(\omega),
$$

Where $R_{*}^{2}(\omega)=c_{* *}\left(1+\rho_{1}^{2 p+2}(\omega)+\rho_{2}^{2}(\omega)\right)$ and $c_{* *}$ is a deterministic positive constant.

Proof Taking the inner product of (4.14) with $v^{b}$, we obtain:

$$
\frac{d}{d t} \phi_{2}(t, \omega)+H_{2}(t, \omega)=0
$$

Where

$$
\phi_{2}(t, \omega)=\frac{1}{2}\left(\left\|v^{b}\right\|^{2}+\alpha\left\|\nabla v^{b}\right\|^{2}+\left\|\eta^{t}\right\|_{\mu, E_{0}}^{2}\right) .
$$

And

$$
H_{2}(t, \omega)=\left\|\nabla v^{b}\right\|^{2}+\left\langle\eta^{t}(s), \eta_{s}^{t}(s)\right\rangle_{\mu, E_{0}}-\left\langle f\left(v+z\left(\vartheta_{t} \omega\right)\right), v^{b}\right\rangle-\left\langle\left(1-\alpha^{2}\right) \nabla z\left(\vartheta_{t} \omega\right), v^{b}\right\rangle-\left\langle\alpha z\left(\vartheta_{t} \omega\right), v^{b}\right\rangle \text {. Note }
$$

that using (1.2)-(1.4) with (4.6) and Yang inequality

$$
\begin{gathered}
\left|f\left(v+z\left(\vartheta_{t} \omega\right)\right), v^{b}\right| \leq\left(\lambda_{1}-w\right)\left\|v+z\left(\vartheta_{t} \omega\right)\right\|\left\|v^{b}\right\| \\
\leq\left(\lambda_{1}-w\right)\left(\frac{\|v\|^{2}}{2}+\left\|v^{b}\right\|^{2}+\frac{\left\|z\left(\vartheta_{t} \omega\right)\right\|^{2}}{2}\right) \\
\leq \frac{\lambda_{1}-w}{2}\|v\|^{2}+\left(1-\frac{w}{\lambda_{1}}\right)\left\|v^{b}\right\|^{2}+\frac{\lambda_{1}-w}{2}\left\|z\left(\vartheta_{t} \omega\right)\right\|^{2} . \\
\left\langle\left(1-\alpha^{2}\right) \nabla z\left(\vartheta_{t} \omega\right), v^{b}\right\rangle+\left\langle\alpha z\left(\vartheta_{t} \omega\right), v^{b}\right\rangle \leq \frac{w}{4 \lambda_{1}}\left\|\nabla v^{b}\right\|^{2}+\frac{\lambda_{1}}{w}\left(1-\alpha^{2}\right)\left\|\nabla z\left(\vartheta_{t} \omega\right)\right\|^{2}+\frac{\lambda_{1}}{w} \alpha\left\|z\left(\vartheta_{t} \omega\right)\right\|^{2} .
\end{gathered}
$$

Then, we can get

$$
\frac{1}{2}\left(\left\|v^{b}\right\|^{2}+\alpha\left\|\nabla v^{b}\right\|^{2}+\left\|\eta^{t}\right\|_{\mu, E_{0}}^{2}\right)+\lambda_{3}\left(\left\|v^{b}\right\|^{2}+\alpha\left\|\nabla v^{b}\right\|^{2}+\left\|\eta^{t}\right\|_{\mu, E_{0}}^{2}\right) \leq c_{1}\left(1+p_{0}\left(\vartheta_{t} \omega\right)\right)+c\|v\|^{2},
$$

Where

$$
\lambda_{3}=\min \left\{\frac{w}{2}, \frac{w}{2 \lambda_{1} \alpha}, \frac{\delta}{2}\right\}
$$

Hence, we have

$$
\frac{d}{d t} \phi_{2}(t, \omega)+\lambda_{3} \phi_{2}(t, \omega) \leq c_{3} p_{0}\left(\vartheta_{t} \omega\right)+c_{4}\left(1+\|v\|^{2}\right)
$$

By Gronwall inequuality, we obtain

$$
\phi_{2}(t, \omega) \leq c_{3} \int_{0}^{t} e^{\lambda_{3}(s-t)} p_{0}\left(\vartheta_{s} \omega\right) d s+c_{4} \int_{0}^{t} e^{\lambda_{3}(s-t)}\|v(s)\|^{2} d s+\frac{1}{\lambda_{3}} c_{4} .
$$

Noeting $\phi_{2}(t, \omega) \geq\left\|Y^{b}(t, \omega)\right\|_{E_{1}}^{2}$ and replacing $\omega$ by $\vartheta_{-t} \omega$, we have 


$$
\begin{gathered}
\left\|Y^{b}\left(t, \vartheta_{-t} \omega\right)\right\|_{E_{1}}^{2} \\
\leq c_{3} \int_{0}^{t} e^{\lambda_{3}(s-t)} p_{0}\left(\vartheta_{s-t} \omega\right) d s+c_{4} \int_{0}^{t} e^{\lambda_{3}(s-t)}\left\|v\left(s, \vartheta_{-t} \omega\right)\right\|^{2} d s+\frac{1}{\lambda_{3}} c_{4} \\
\leq c_{3} \int_{-t}^{0} e^{\lambda_{3} s} p_{0}\left(\vartheta_{s} \omega\right) d s+c_{4} \int_{0}^{t} e^{\lambda_{3}(s-t)}\left\|v\left(s, \vartheta_{-t} \omega\right)\right\|^{2} d s+\frac{1}{\lambda_{3}} c_{4} .
\end{gathered}
$$

By Corollary 3.2. the first term on the rirht hand side of () satixfies

$$
c_{3} \int_{-t}^{0} e^{\lambda_{3} s} p_{0}\left(\vartheta_{s} \omega\right) d s \leq c_{3} \int_{-t}^{0} e^{\frac{1}{2} \lambda_{3} s} \rho_{2}^{2}(\omega) d s \leq \frac{2}{\lambda_{3}} c_{6} \rho_{2}^{2}(\omega) \text { for } t \geq 0
$$

By (4.12), we can get

$$
\left\|v\left(s, \vartheta_{-t} \omega\right)\right\|^{2} \leq c_{0}\left\|Y\left(\vartheta_{-t} \omega\right)\right\|_{E_{0}}^{2} e^{-\lambda_{3} s}+c_{*}\left(1+\rho_{1}^{2 p+2}(\omega)+\rho_{2}^{2}(\omega)\right)
$$

Thus, the second term on the right hand side of () satisfies for $t \geq 0$

$$
c_{4} \int_{0}^{t} e^{\lambda_{3}(s-t)}\left\|v\left(s, \vartheta_{-t} \omega\right)\right\|^{2} d s \leq c_{0} c_{4} t e^{-\lambda_{3} t}\left\|Y\left(\vartheta_{-t} \omega\right)\right\|_{E_{0}}^{2}+\frac{c_{4}}{\lambda_{3}} c_{*}\left(1+\rho_{1}^{2 p+2}(\omega)+\rho_{2}^{2}(\omega)\right) .
$$

Let $R_{*}^{2}(\omega)=\frac{1}{\lambda_{3}}\left(2 c_{3}+c_{4}+c_{4} c_{*}\right)\left(1+\rho_{1}^{2 p+2}(\omega)+\rho_{2}^{2}(\omega)\right)$. It follows from (4.22)-(4.25) that

$$
\left\|Y^{b}\left(t, \vartheta_{-t} \omega\right)\right\|_{E_{1}}^{2} \leq c_{0} c_{4} t e^{-\lambda_{3} t}\left\|Y\left(\vartheta_{-t} \omega\right)\right\|_{E_{0}}^{2}+R_{*}^{2}(\omega)
$$

Since $\{B(\omega)\}_{\omega \in \Omega} \in D$ is tempered and $Y_{0}\left(\vartheta_{-t} \omega\right) \in B\left(\vartheta_{-t} \omega\right) \eta^{t}$, there exist $T_{B}^{*}(\omega)>0$ such that for $t \geq T_{B}^{*}(\omega)$.

$$
c_{0} c_{4} t e^{-\lambda_{3} t}\left\|Y\left(\vartheta_{-t} \omega\right)\right\|_{E_{0}}^{2} \leq R_{*}^{2}(\omega)
$$

Thus

$$
\left\|Y^{b}\left(t, \vartheta_{-t} \omega, Y_{0}\left(\vartheta_{-t} \omega\right)\right)\right\|_{E_{0}}^{2} \leq 2 R_{*}^{2}(\omega)
$$

And the result holds.

We are now in a position to present our main result:

- Theorem 4.4 Assume (1.4) hold. Then the random dynamic system $S(t, \omega)$ has a unique random attractor in $E_{0}$.

- Proof. By Lemma 2.4, Lemma 4.2 and Lemma 4.3 , the stochastic dynamical system $S(t, \omega)$ of the nonlinear is almost surely $D-\alpha$-contracting. This together with Lemma2.5 implies that the existence of a unique $D$ - random attractor for $S(t, \omega)$.

\section{REFERENCES}

1. Aifantis EC. On the problem of diffusion in solids, Acta Mech.1980; 37: 265-296.

2. Borini S, Pata V. Uniform attractors for a strongly damped wave equation with linear memory. Asymptotic Analysis. 1999 Jan 1;20(3- 4):263-77.

3. Dafermos CM. Asymptotic stability in viscoelasticity, Arch. Ration. Mech. Anal. 37
(1970) 297-308.

4. Gatti S, Miranville A, Pata V, Zelik S. Attractors for semi-linear equations of viscoelasticity with very low dissipation. The Rocky Mountain Journal of Mathematics. 2008 Jan 1:1117-38.

5. Giorgi C, Rivera JE, Pata V. Global attractors for a semilinear hyperbolic equation in viscoelasticity. Journal of Mathematical Analysis and Applications. 2001 Aug 1;260(1):83-99.

6. Lions JL, Magenes E. Non-homogeneous boundary value problems and applications. Springer Science \& Business Media; 2012 Dec 6.

7. Chen PJ, Williams WO. A note on non-simple heat conduction. Zeitschrift für angewandte Mathematik und Physik ZAMP. 1968 Nov 1;19(6):969-70.

8. Pata V, Zucchi A. Attractors for a damped hyperbolic equation with linear memory.2001.

9. Sun CY, Wang SY, Zhong CK. Global attractors for a nonclassical diffusion equation. Acta Mathematica Sinica, English Series. 2007 Jul 1;23(7):1271-80.

10. Sun C, Yang M. Dynamics of the nonclassical 
diffusion equations. Asymptotic Analysis. 2008 Jan 1;59(1-2):51-81.

11. Temam R. Infinite-dimensional dynamical systems in mechanics and physics. Springer Science \& Business Media; 2012 Dec 6.

12. Wang S, Li D, Zhong C. On the dynamics of a class of nonclassical parabolic equations. Journal of Mathematical Analysis and Applications. 2006 May 15;317(2):565-82.

13. Xiao YL. Attractors for a nonclassical diffusion equation. Acta Mathematicae Applicatae Sinica. 2002 Jun 1;18(2):273-6.

14. Wang X, Yang L, Zhong C. Attractors for the nonclassical diffusion equations with fading memory. Journal of mathematical analysis and applications. 2010 Feb 15;362(2):327-37.

15. Caraballo T, Langa JA, Robinson JC. A stochastic pitchfork bifurcation in a reaction-diffusion equation. Proceedings of the Royal Society of London. Series A: Mathematical, Physical and Engineering Sciences. 2001 Sep 8;457(2013):204161.

16. Crauel H, Debussche A and F. Flandoli, Random attractors, J. Dynam. Differential Equations, 9(1997), 307-341.372 X. Du

17. Zhengde D, Xianyun D. Global attractor for the nonlinear strain waves in elastic waveguides. Acta Mathematicae Applicatae Sinica. 2001 Apr $1 ; 17(2): 260-70$.

18. Debussche A. Hausdorff dimension of a random invariant set. Journal de mathématiques pures et appliquées. 1998 Dec 1;77(10):967-88.

19. Deimling K. Nonlinear functional analyis, Springer, Berlin; 1985.

20. Lv Y, Wang W. Limiting dynamics for stochastic wave equations. Journal of Differential Equations. 2008 Jan 1;244(1):1-23.

21. Scheutzow M. Comparison of various concepts of a random attractor:I A case study. Archiv der Mathematik. 2002 Mar 1;78(3):233-40.

22. Zhou S. Dimension of the global attractor for strongly damped nonlinear wave equation. Journal of mathematical analysis and applications. 1999 May 1;233(1):102-15.

23. Temam R. Infifinite-Dimensional Systems in Mechanics and Physics, New York, SpringerVerlag; 1988.

24. Du X. Random Attractor of Nonlinear Strain Waves with White Noise. Journal of Applied Analysis and Computation. 2011 Jan 1;1(3):36172. 